Supplement of Earth Surf. Dynam., 9, 1279-1300, 2021

https://doi.org/10.5194/esurf-9-1279-2021-supplement

(C) Author(s) 2021. CC BY 4.0 License.

(c) (i)

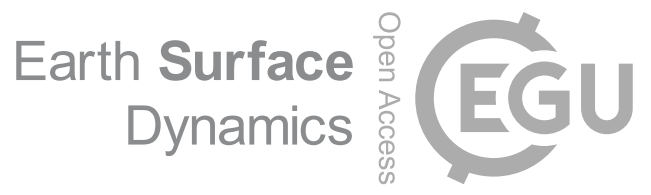

Supplement of

\title{
Hilltop curvature as a proxy for erosion rate: wavelets enable rapid computation and reveal systematic underestimation
}

\author{
William T. Struble and Joshua J. Roering \\ Correspondence to: William T. Struble (wtstruble@ arizona.edu)
}

The copyright of individual parts of the supplement might differ from the article licence. 
a)

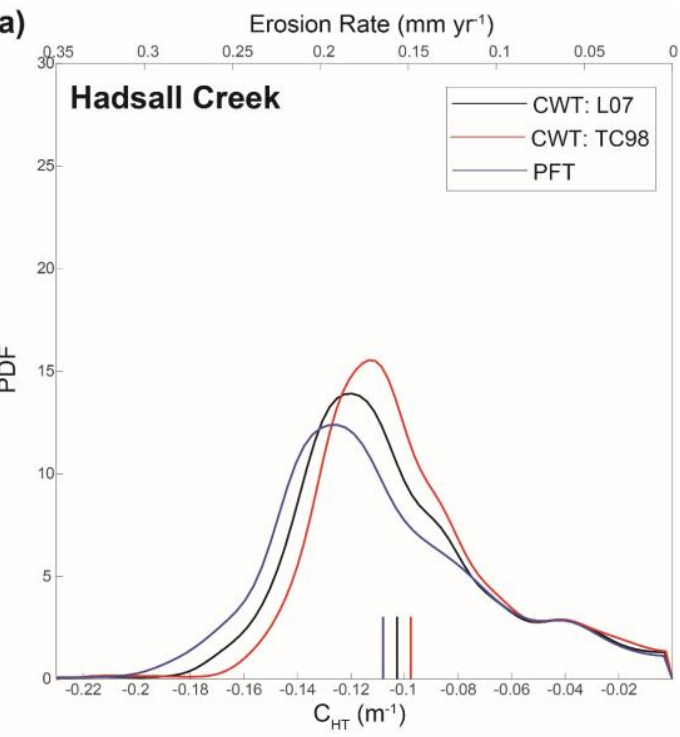

b)

Erosion Rate $\left(\mathrm{mm} \mathrm{yr}^{-1}\right)$

N. Fork Smith River

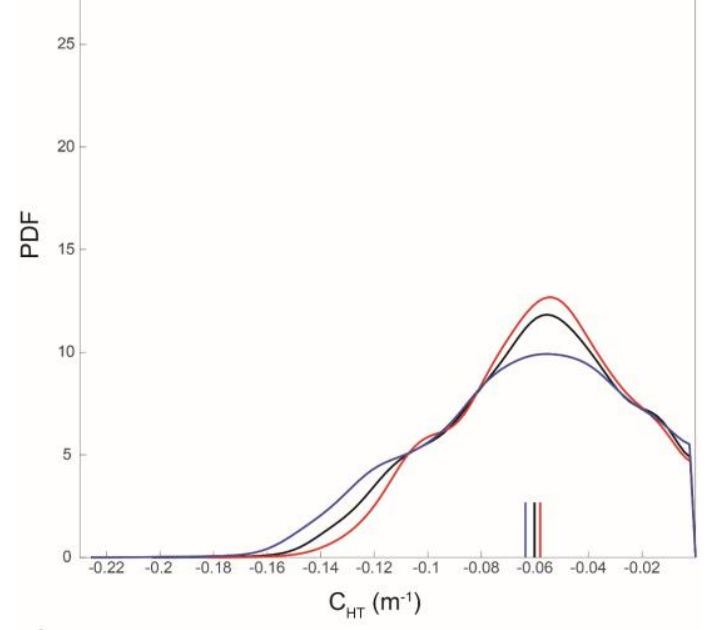

c)

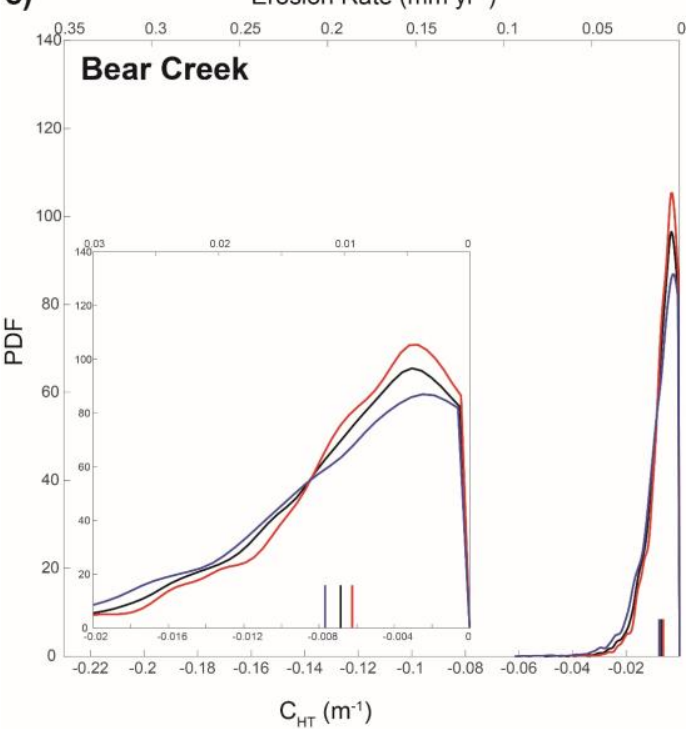

Figure S1: Probability density functions of $\mathrm{C}_{\text {HT-P }}$ and $\mathrm{C}_{\mathrm{HT}-\mathrm{w}}$ and erosion rate calculated using Equation 1 for all mapped hilltops at each OCR field site. See Figure 5 for representative hilltop PDFs for each catchment. Note agreement between each $\mathrm{CHT}_{\text {HT }}$ measurement technique. Further, note dramatic variability in $\mathrm{C}_{\text {Hт }}$ between all site (all panels use same $\mathrm{x}$-axis; inset in panel $\mathrm{C}$ more clearly displays distribution of $\mathrm{C}_{\mathrm{HT}}$ at Bear Creek). Small vertical lines at bottom of each panel represent the mean of the plotted distribution (Table 2). Note that positive $C_{\text {HT }}$ values are not permitted in the output PDF. 

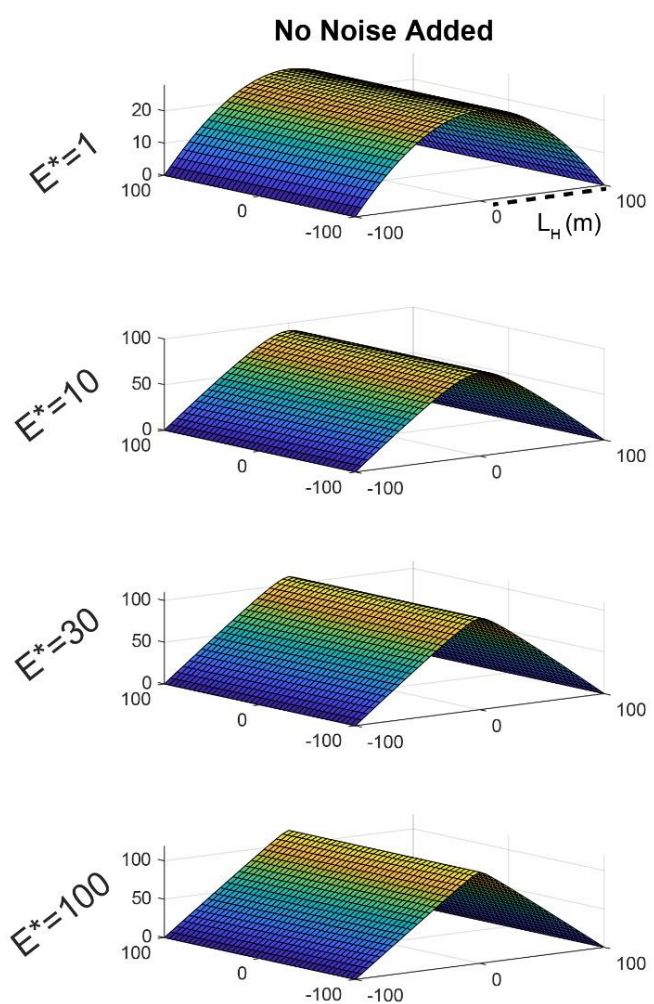

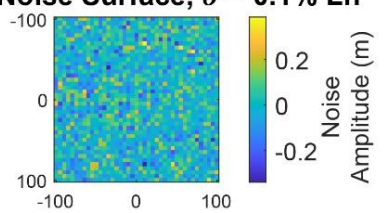

White Noise Surface, $\sigma=\mathbf{0 . 5} \% \mathrm{Lh}$

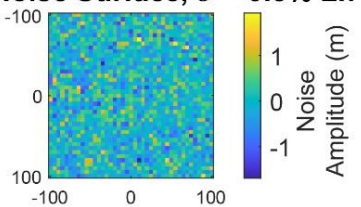

White Noise, $\sigma=0.1 \%$ Lh
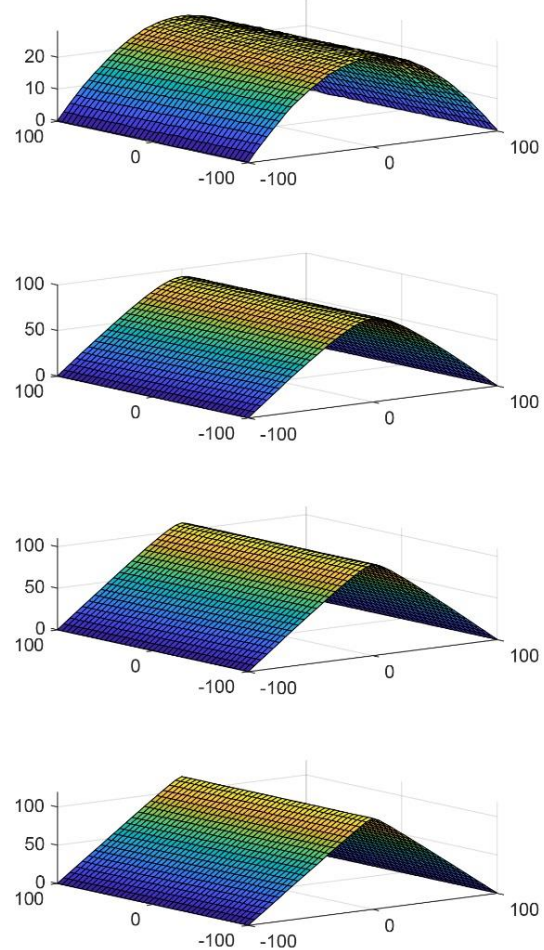

White Noise, $\sigma=0.5 \%$ Lh
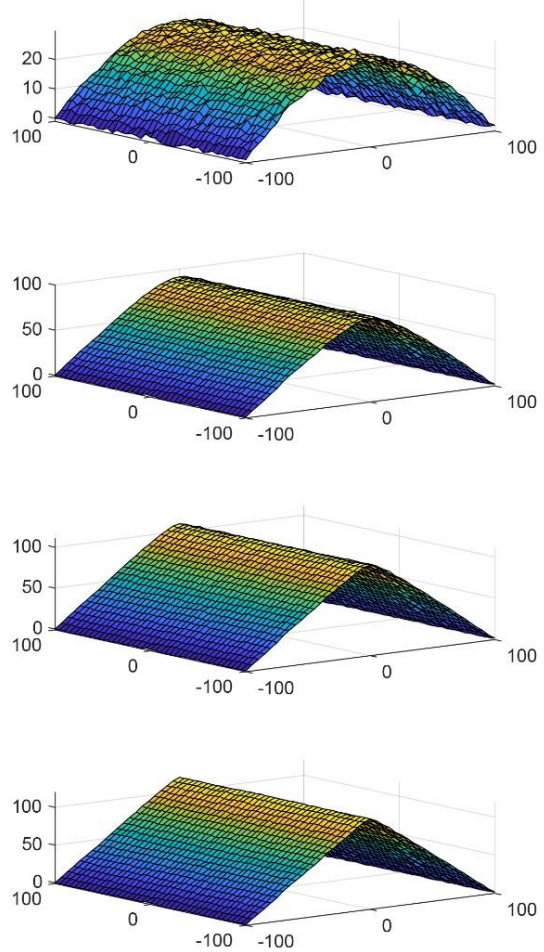

White Noise Surface, $\sigma=\mathbf{5} \%$ Lh

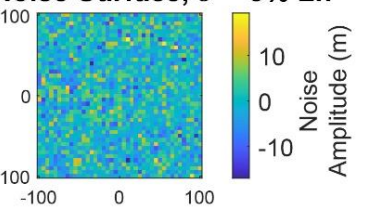

White Noise, $\sigma=5 \%$ Lh
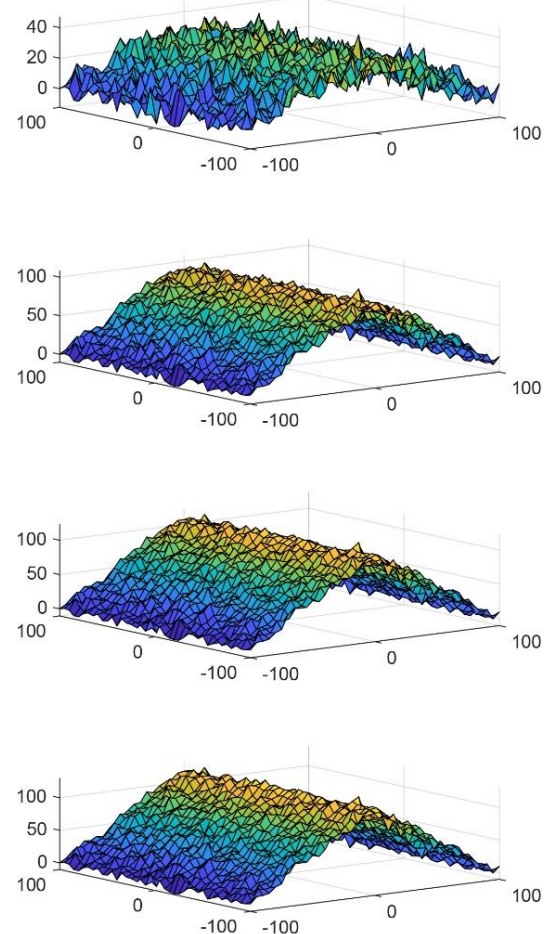

Figure S2: Synthetic hillslopes constructed using Equation 9. Upper row shows white noise surfaces that are added to the original hillslope form (left column); yellow colors correspond with positive deviations from the hillslope (convex noise) and blue with negative deviations (concave noise). Each row of hillslopes

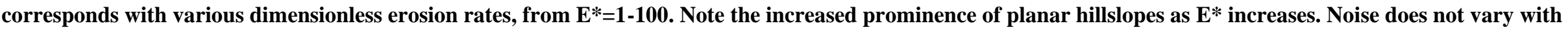

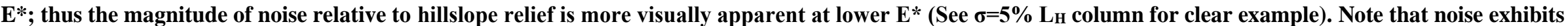
little randomness at long-wavelengths, which is apparent in the pink and red noise hillslopes in Figures 7 and 8. 

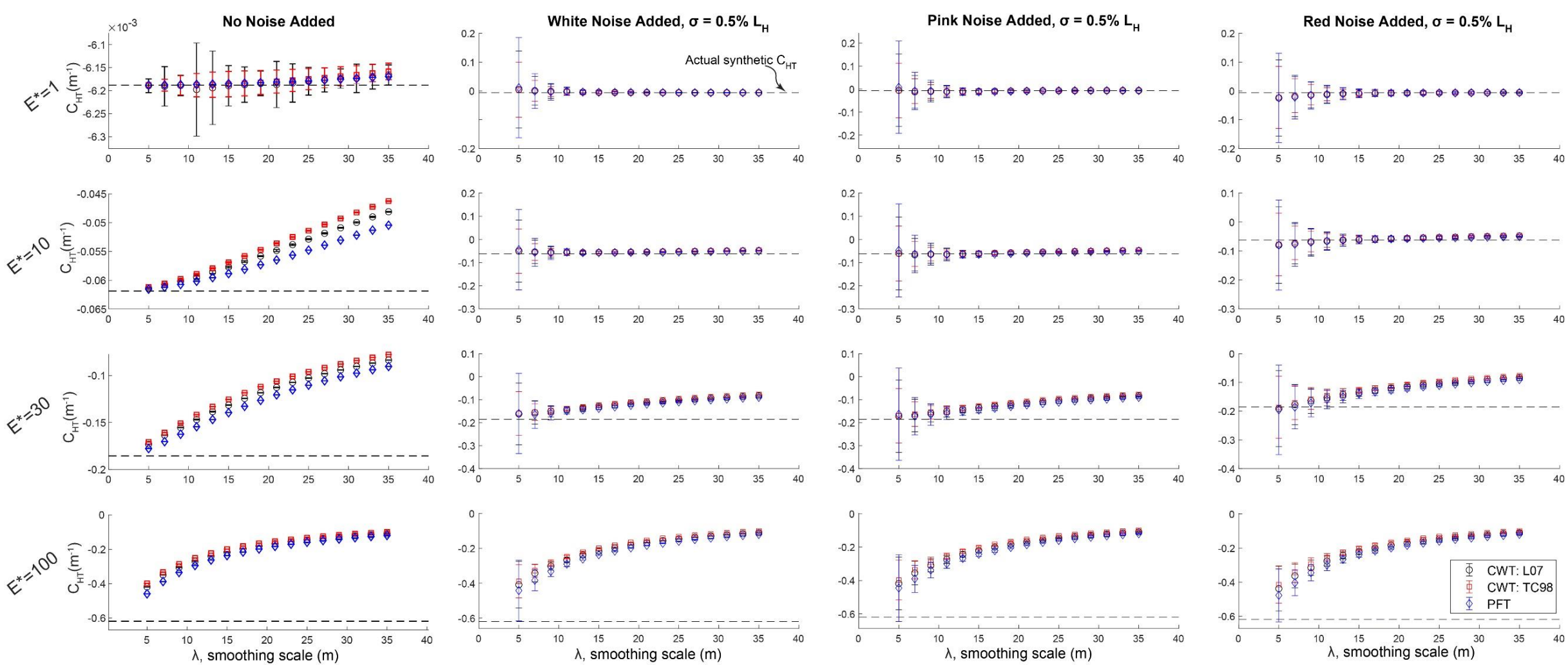

Figure S3: Hilltop curvature when noise amplitude $\sigma=0.5 \% \mathrm{LH}_{\mathrm{H}} \mathrm{E}^{*}$ increases from $\mathrm{E}^{*}=1$ in top row to $\mathrm{E}^{*}=100$ in bottom row. Left column corresponds to synthetic hillslope is no added noise, while columns 2-4 correspond with hillslopes where white, pink, and red noise have been added, respectively. Error bars are $1 \sigma$ standard deviation on the hilltop. Dashed black line is model-specified $\mathbf{C}_{\mathrm{HT}}$ for the synthetic hillslope. For clarity of deviation between measured and known $\mathrm{C}_{\mathrm{HT}}$, see the ratio of the two values in Figure 9. 

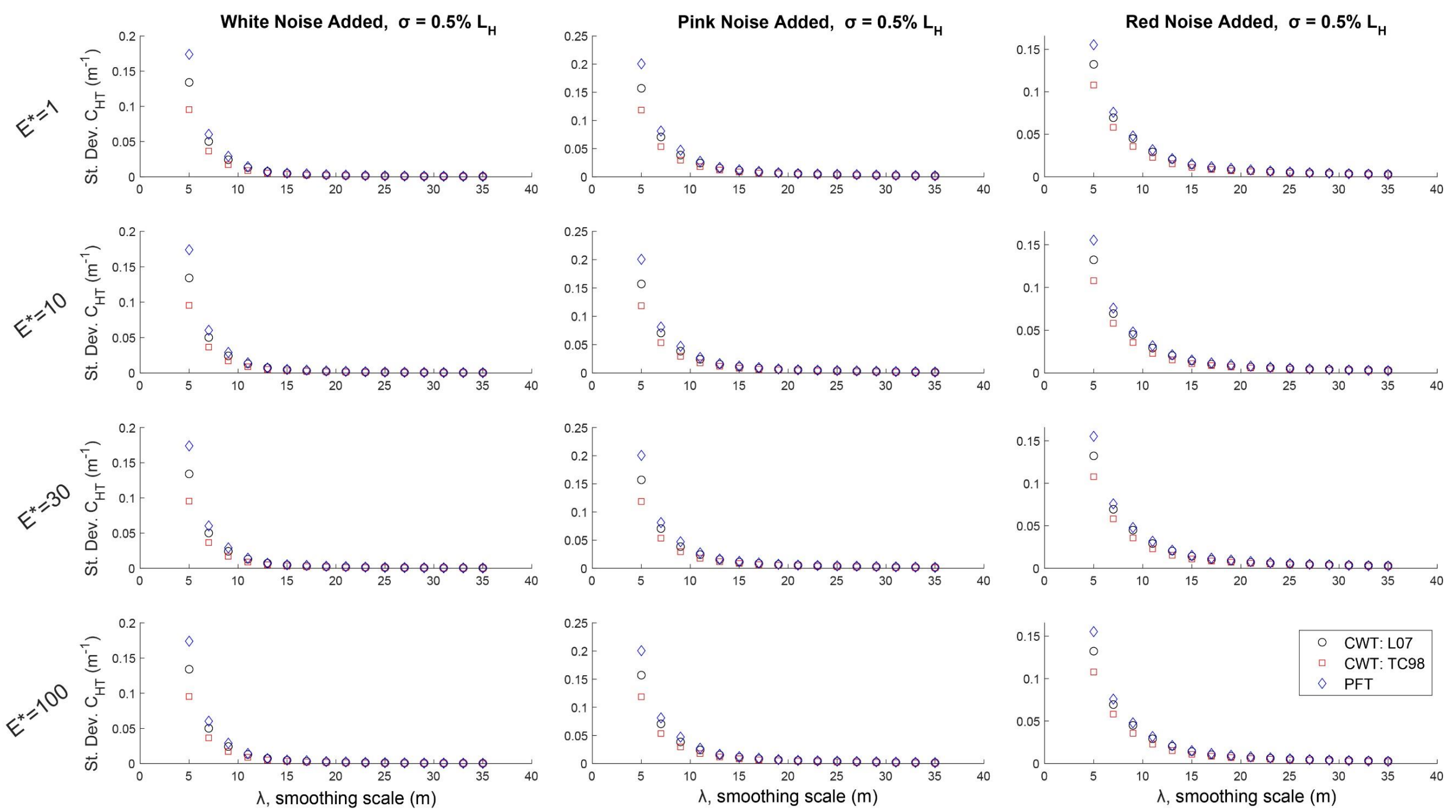

Figure S4: Standard deviation of $\mathrm{C}_{\mathrm{HT}}$ for noise amplitude $\sigma=0.5 \% \mathrm{~L}_{\mathrm{H}}$. These values correspond to the error bars in Figure S3. Note that surface noise does not vary as a function of $E^{*}$. Therefore, given the distributive property of convolutions (see main text for details), the standard deviation does not vary with $E^{*}$. Note that the standard deviation is high at small smoothing scales when the signal to noise ratio is high. As topography is smoothed, the uncertainty in $\mathrm{C}_{\mathrm{HT}}$ along the hilltop decreases. 

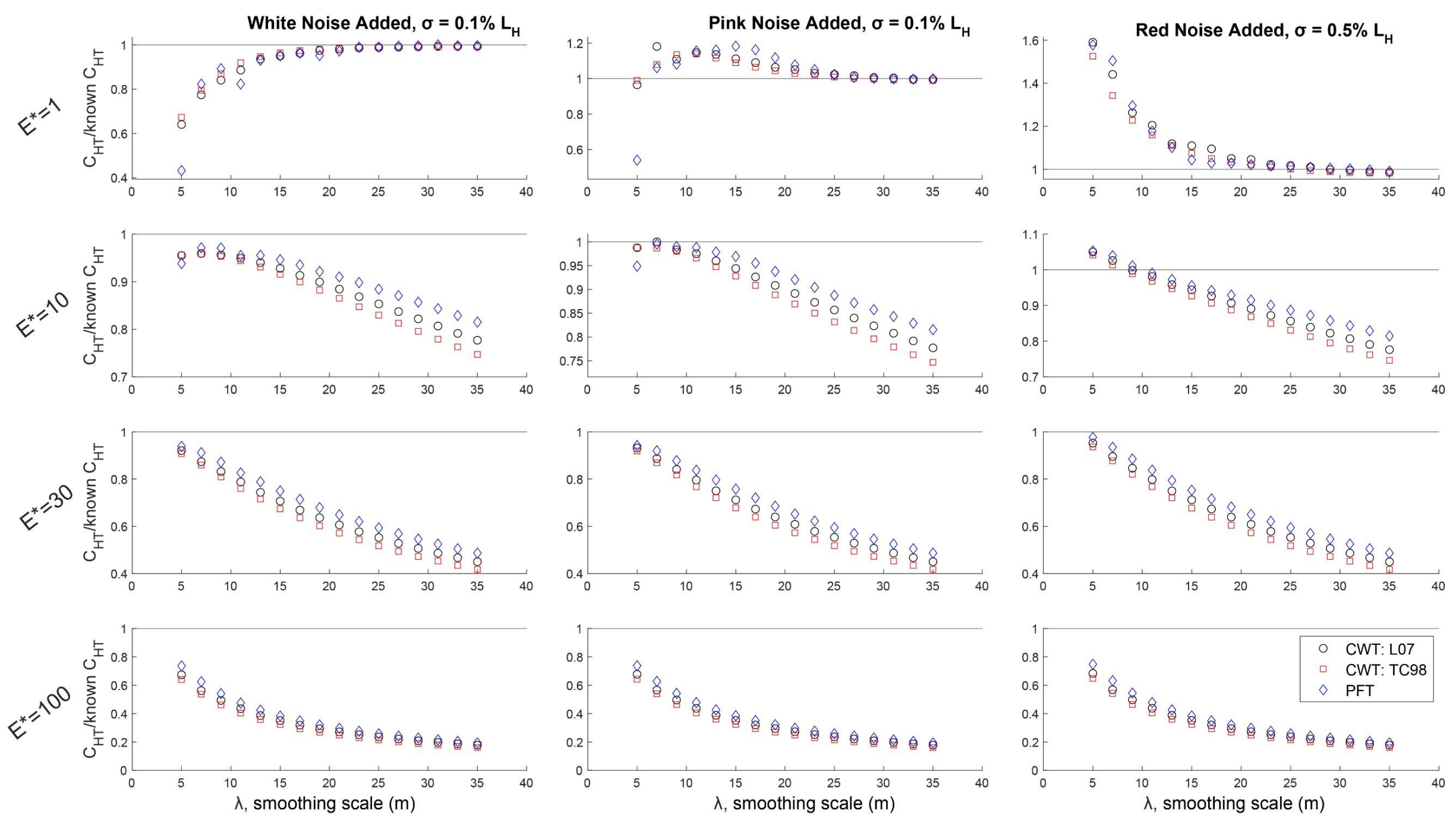

Figure S5: Ratio of $\mathrm{C}_{H T}$ of synthetic hillslopes where $E^{*}=1,10,30$, and 100 measured at various smoothing scales, $\lambda$, with: $\sigma=0.1 \% L_{H}$ white noise (first column), pink noise (second column), and red (Brownian) noise (third column). Ratio of $\mathrm{C}_{\mathrm{HT}}$ is quantified as the quotient of the $\mathrm{C}_{\mathrm{HT}}$ measured with the CWT or PFT and the actual synthetic $\mathbf{C}_{\text {Hт. }}$ Black horizontal line in each panel corresponds with where the measured $\mathbf{C}_{\text {HT }}$ equals the actual synthetic $\mathbf{C}_{\mathrm{HT}}$ (i.e. ratio=1). Points that plot above the line correspond with locations where $\mathrm{C}_{\mathrm{HT}}$ is overestimated; points that plot below are underestimations. Note that compared to Figure 9 ( $\sigma=0.5 \% \mathrm{LH}_{\mathrm{H}}$, deviation between the measured and actual synthetic $C_{\mathbf{H T}}$ is less prominent at slow erosion rates $\left(E^{*}=1-10\right)$, though $E^{*}$ is still underestimated when $E^{*}=10$. This is not unexpected given the underestimation of $E^{*}$ when no noise is added to the surface (see first column in Figure 9). 

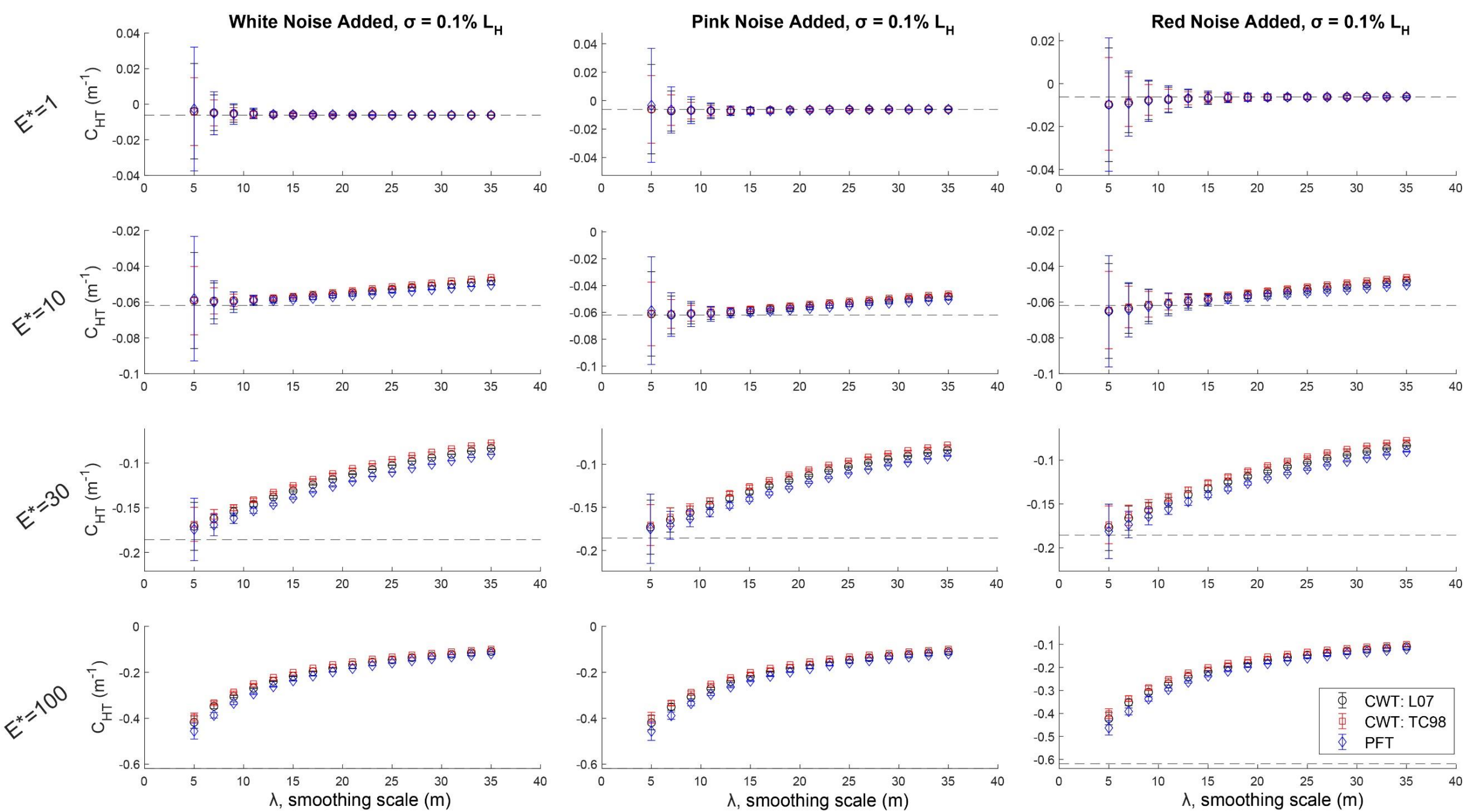

Figure S6: Hilltop curvature when noise amplitude $\sigma=0.1 \% \mathrm{~L}_{\mathrm{H}} . \mathrm{E}^{*}$ increases from $\mathrm{E}^{*}=1$ in top row to $\mathrm{E}^{*}=100$ in bottom row. Left column corresponds to synthetic hillslope is no added noise, while columns 2-4 correspond with hillslopes where white, pink, and red noise have been added, respectively. Error bars are $1 \sigma$ standard deviation on the hilltop. Dashed black line is known $\mathbf{C}_{\mathrm{HT}}$ from the synthetic hillslope. For clarity of deviation between measured and known $\mathrm{C}_{\mathrm{HT}}$, see the ratio of the two values in Figure S5. 

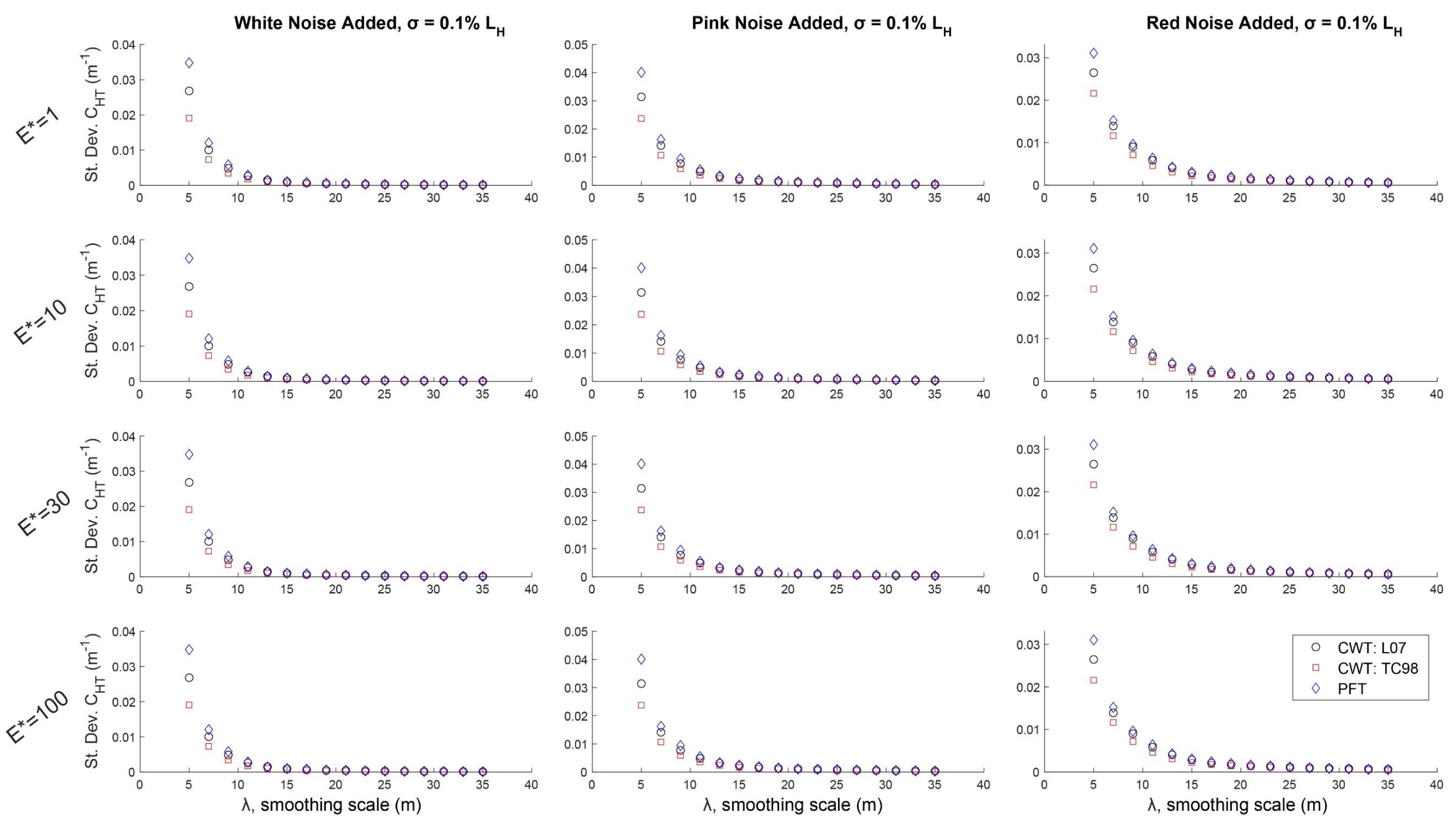

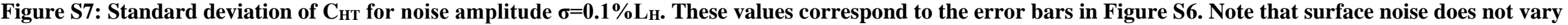

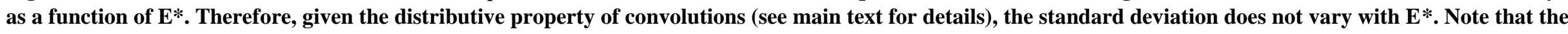
standard deviation is high at small smoothing scales when the signal to noise ratio is high. As topography is smoothed, the uncertainty in CHT along the hilltop decreases. 

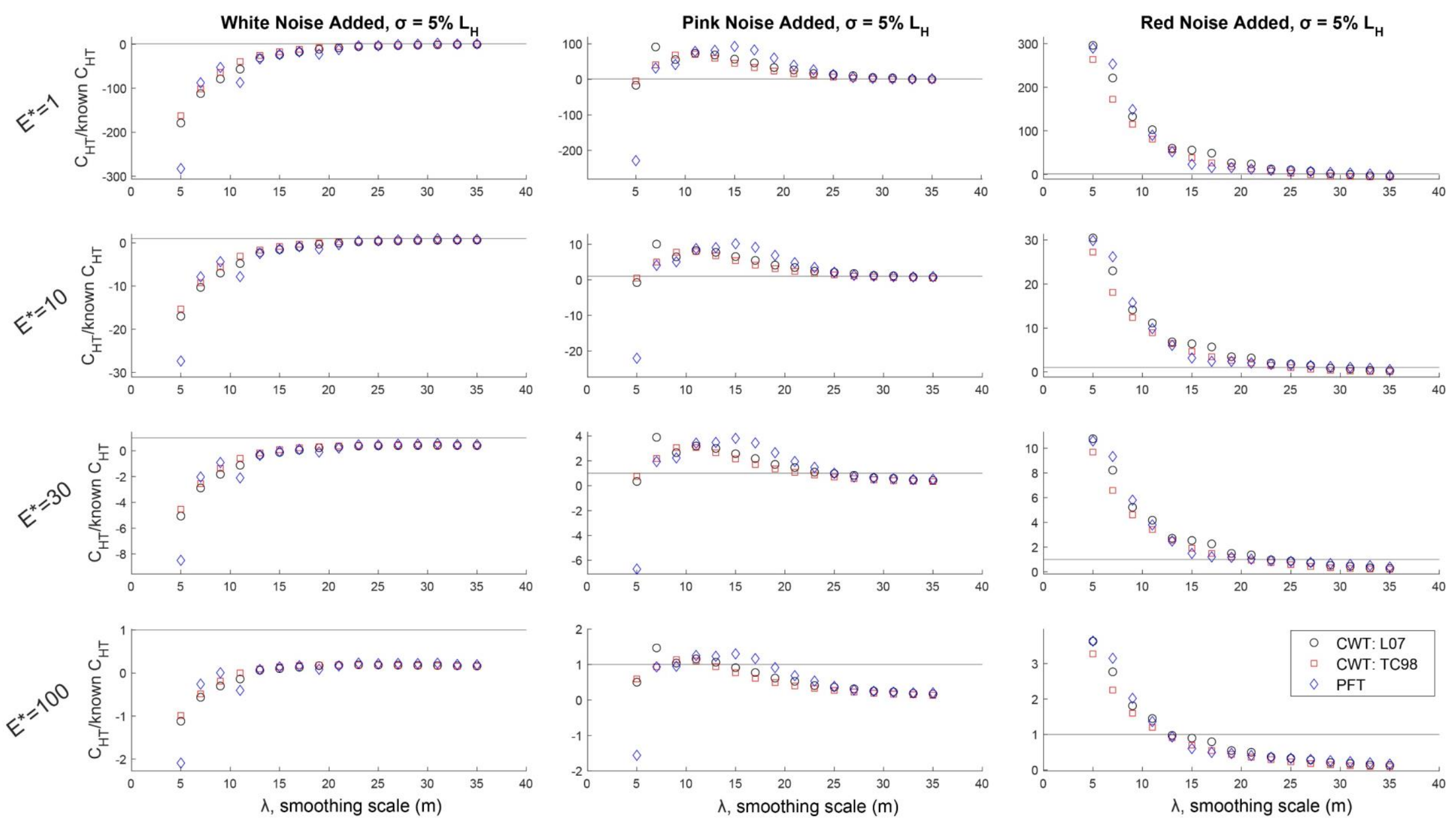

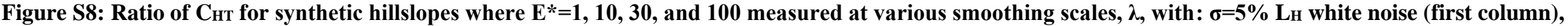

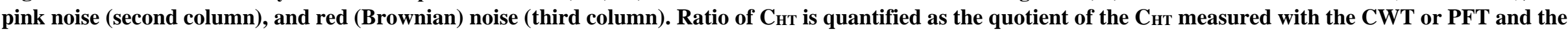

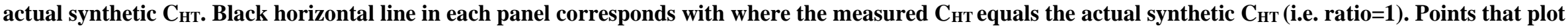

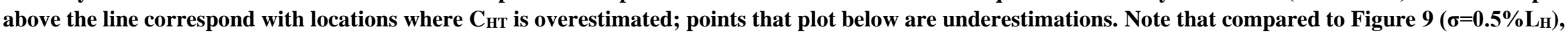

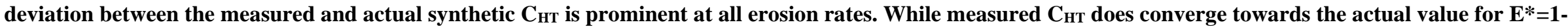

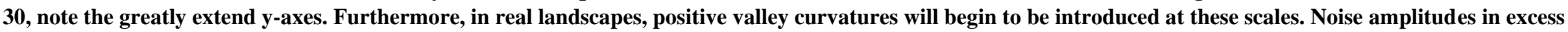
of $5 \mathbf{~ m}$ are likely unphysical in real landscapes. However, this case demonstrates that noise remains a hindrance for curvature measurement techniques as

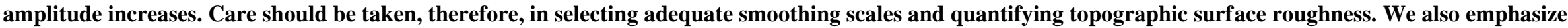

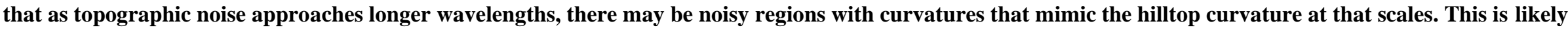
rare, but would depend on the measurement scale and, in the synthetic case, the iteration of noise generation. 

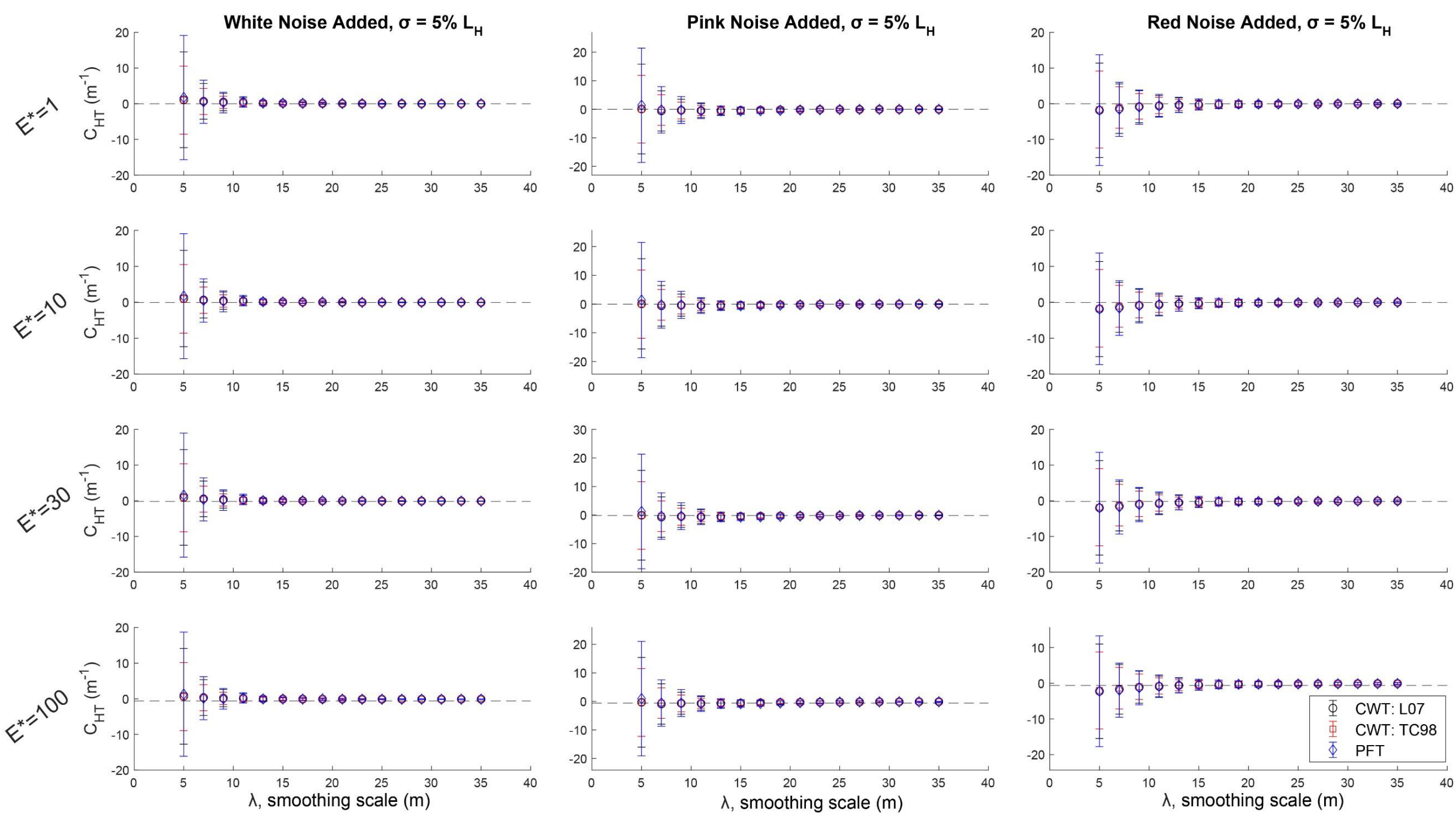

Figure S9: Hilltop curvature when noise amplitude $\sigma=5 \% \mathrm{LH} . \mathrm{E}^{*}$ increases from $\mathrm{E}^{*}=1$ in top row to $\mathrm{E}^{*}=100$ in bottom row. Left column corresponds to synthetic hillslope is no added noise, while columns 2-4 correspond with hillslopes where white, pink, and red noise have been added, respectively. Error bars are $1 \sigma$ standard deviation on the hilltop. Dashed black line is known $\mathrm{C}_{\mathrm{HT}}$ from the synthetic hillslope. For clarity of deviation between measured and known $\mathrm{C}_{\mathrm{HT}}$, see the ratio of the two values in Figure S8. 

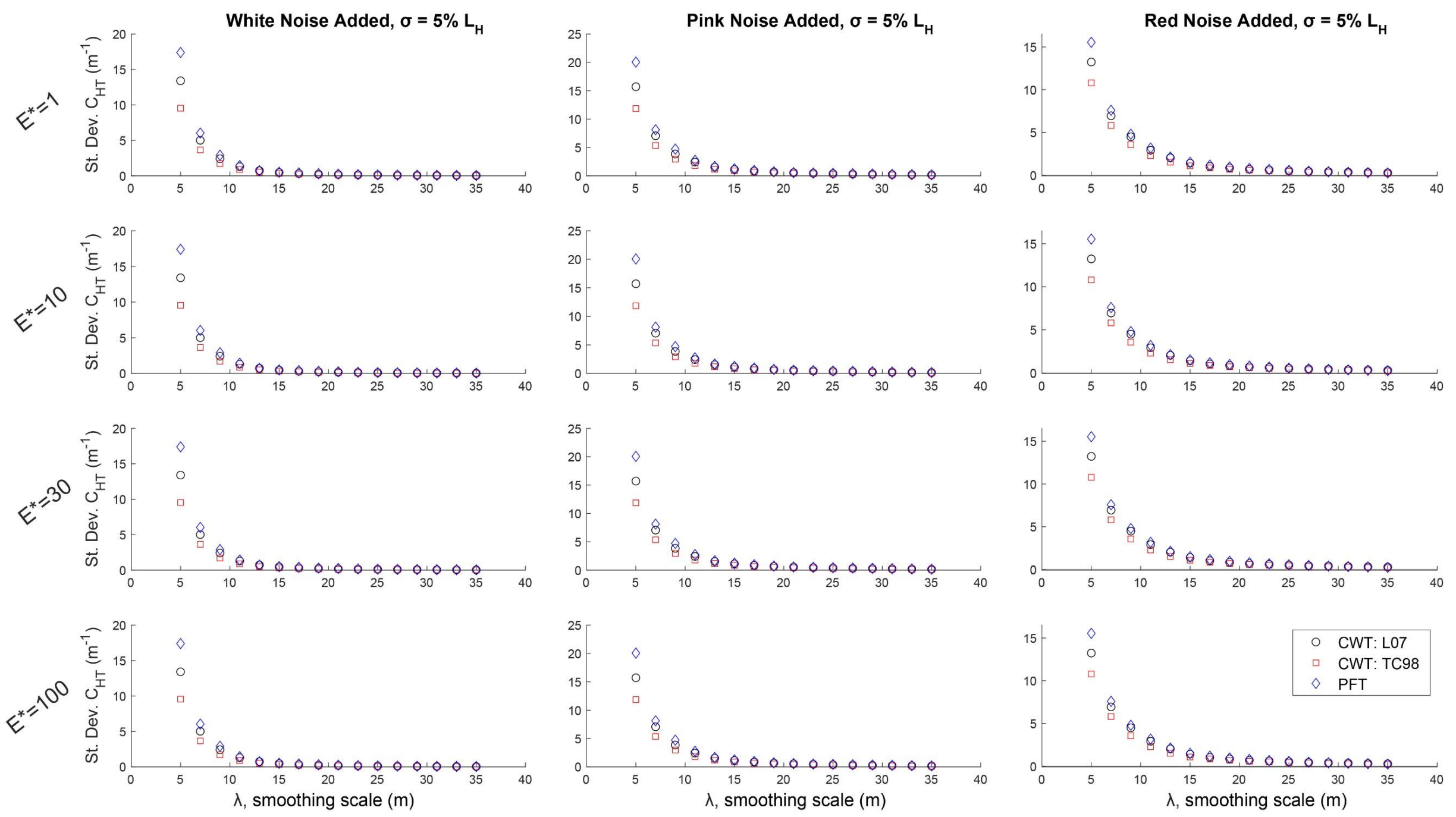

Figure S10: Standard deviation of $\mathrm{C}_{\mathrm{HT}}$ for noise amplitude $\sigma=\mathbf{5 \%} \mathrm{L}_{\mathrm{H}}$. These values correspond to the error bars in Figure S9. Note that surface noise does not vary as a function of $E^{*}$. Therefore, given the distributive property of convolutions (see main text for details), the standard deviation does not vary with $E^{*}$. Note that the standard deviation is high at small smoothing scales when the signal to noise ratio is high. As topography is smoothed, the uncertainty in $\mathrm{C}_{\mathrm{HT}}$ along the hilltop decreases. Despite the high magnitude of the surface noise, the CWT and PFT still manage to output relatively consistent along-hilltop curvatures at larger smoothing scales. 